\title{
ON ENTROPY AND GENERATORS OF MEASURE-PRESERVING TRANSFORMATIONS
}

\author{
BY \\ WOLFGANG KRIEGER
}

\begin{abstract}
Let $T$ be an ergodic measure-preserving transformation of a Lebesgue measure space with entropy $h(T)$. We prove that $T$ has a generator of size $k$ where $e^{h(T)} \leqq k \leqq e^{h(T)}+1$.
\end{abstract}

1. Introduction. In this paper we are concerned with ergodic invertible measurepreserving transformations of a Lebesgue measure space $(E, \mathfrak{B}, p)$. By a partition $\left\{A_{n}: n \in \theta\right\}$ of $E$ we shall mean a finite or countably infinite collection of disjoint sets $A_{n} \in \mathfrak{B}$ of positive measure such that

$$
E=\bigcup_{n \in \theta} A_{n}
$$

We call a partition $\left\{A_{n}: n \in \theta\right\}$ a generator of an i.m.p.t. $T$ of $(E, \mathfrak{B}, p)$ if $\mathfrak{B}$ is generated by

$$
\bigcup_{i=-\infty}^{\infty}\left\{T^{i} A_{n}: n \in \theta\right\}
$$

For the theory of entropy and generators of i.m.p.t. we refer to [1], [4], [5] and [6]. It was proved by V. A. Rohlin that every aperiodic i.m.p.t. with finite entropy has a generator with finite entropy $[6,10.7]$. We shall prove in $\$ 2$ that every ergodic i.m.p.t. with finite entropy has a finite generator, thereby solving a problem that was posed by V. A. Rohlin [6, p. 30].

Throughout most of this paper we shall be given a finite or countably infinite state space $\Omega$. For finite $\Omega$ we shall prove in $\S 3$ an approximation theorem for probability measures on $\Omega^{Z}$ that are invariant under the shift $S$,

$$
(S x)_{i}=x_{i+1}, \quad i \in Z, \quad x=\left(x_{i}\right)_{i=-\infty}^{\infty} \in \Omega^{z} .
$$

This theorem will enable us to derive in $\S 4$ from the work of A. H. Zaslavskir [7] a formula for the minimal number of elements that a generator of an ergodic i.m.p.t. can contain. Denote this number by $\Delta(T)$. If the entropy $h(T)$ of $T$ is infinite then $\Delta(T)$ is also infinite, if $h(T)<\infty$, then $\Delta(T) \geqq e^{h(T)}$. Our result is

$$
\Delta(T) \leqq e^{h(T)}+1 .
$$

Received by the editors March 18, 1969.

AMS Subject Classifications. Primary 2870; Secondary 5482.

Key Words and Phrases. Ergodic measure-preserving transformations, entropy, generators of ergodic measure-preserving transformations, shift-invariant measures, coding.

Copyright (C) 1970, American Mathematical Society 
This answers for the ergodic case another question raised by Rohlin [6, p. 30]. In particular it follows that every ergodic i.m.p.t. with entropy zero has a generator with two elements. This was known before in the case of the quasi-discrete spectrum [3, p. 187].

\section{The existence of finite generators.}

(2.1) THEOREM. Every ergodic i.m.p.t. with finite entropy has a finite generator.

Proof. 1. Let $\left\{A_{n}: n \in N\right\}$ be a partition of $(E, \mathfrak{B}, p)$ with finite entropy. Then there exists a mapping $n \rightarrow K_{n} \in N(n \in N)$ and a 1-1 mapping

$$
\varphi: N \rightarrow \bigcup_{k=1}^{\infty}\{1,2,3\}^{k}
$$

where $\varphi(n) \in\{1,2,3\}^{K_{n}}, n \in N$, such that

$$
\sum_{n=1}^{\infty} K_{n} p\left(A_{n}\right)<\infty
$$

For a proof of this let $p\left(A_{n}\right) \geqq p\left(A_{n+1}\right), n \in N$, and let $l(n), n \in N$, be nonnegative integers such that

$$
-\log p\left(A_{n}\right)-1<l(n) \leqq-\log p\left(A_{n}\right), \quad n \in N .
$$

Let further

$$
n_{1}=1, \quad n_{m}=\min \left\{n>n_{m-1}: l(n)>l\left(n_{m-1}\right)\right\}, \quad m>1 .
$$

Then

$$
\sum_{m=1}^{\infty}\left(n_{m+1}-n_{m}\right) 3^{-l\left(n_{m}\right)}=\sum_{n=1}^{\infty} 3^{-l(n)}
$$

and we see from (2) that

$$
\sum_{m=1}^{\infty}\left(n_{m+1}-n_{m}\right) 3^{-l\left(n_{m}\right)} \leqq e
$$

Consequently, for some $m_{0} \in N$,

$$
n_{m+1}-n_{m}<3^{l\left(n_{m}\right)}, \quad m \geqq m_{0} .
$$

We set $K_{n}=l(n), n \geqq n_{m_{0}}$. By (3) it is possible to assign to every $n \geqq n_{m_{0}}$ an element $\varphi(n)$ of $\{1,2,3\}^{K_{n}}$ such that $n \rightarrow \varphi(n), n \geqq n_{m_{0}}$, is $1-1$. The inequality (3) also shows that it is possible to define the $\varphi(n) \in \bigcup_{k=1}^{\infty}\{1,2,3\}^{k}, 1 \leqq n<n_{m_{0}}$, in such a way that

$$
\varphi: N \rightarrow \bigcup_{k=1}^{\infty}\{1,2,3\}^{k}
$$

is 1-1. In order to show that (1) holds it suffices to show that

$$
\sum_{n=n_{m_{0}}}^{\infty} K_{n} p\left(A_{n}\right)<\infty,
$$


and this follows from the finiteness of the entropy of $\left\{A_{n}: n \in N\right\}$ and from (2):

$$
\sum_{n=n_{m_{0}}}^{\infty} K_{n} p\left(A_{n}\right) \leqq \sum_{n=1}^{\infty} l(n) p\left(A_{n}\right) \leqq \sum_{n=1}^{\infty}-p\left(A_{n}\right) \log p\left(A_{n}\right)<\infty .
$$

2. Let $\Omega$ be a finite set containing more than two elements. Let $\omega \in \Omega, C \in N$, and let

$$
\begin{array}{r}
X=\left\{x=\left(\left(x_{i, j}\right)_{j=1}^{D_{i}}\right)_{i=-\infty}^{\infty} \in\left(\bigcup_{k=2}^{\infty} \Omega^{k}\right)^{Z}:\right. \\
\left.x_{i, j} \neq \omega, 1 \leqq j<D_{i}, x_{i, D_{i}}=\omega,-\infty<i<\infty\right\},
\end{array}
$$

and

$$
X_{C}=\bigcap_{k=-\infty}^{\infty} \bigcup_{l=1}^{\infty}\left\{x=\left(\left(x_{i, j}\right)_{j=1}^{D_{i}}\right)_{i=-\infty}^{\infty} \in X: \sum_{m=k}^{k+l}\left(D_{m}-C\right) \leqq 0\right\} .
$$

We are going to construct a 1-1 Borel mapping $U: X_{C} \rightarrow\left(\Omega^{C}\right)^{Z}$ that commutes with the shifts.

Let $x=\left(\left(x_{i, j}\right)_{j=1}^{D_{i}}\right)_{i=-\infty}^{\infty} \in X_{C}$ and let $\Gamma=\left\{i \in Z: D_{i}>C\right\}$. We define for $i \in \Gamma$, $C<j \leqq D_{i}$,

$$
\begin{gathered}
I(i, j)=\min \left\{l>i: j-C+\sum_{i<m \leqq l}\left(D_{m}-C\right) \leqq 0\right\}, \\
J(i, j)=j+\sum_{i<m \leqq I(i, j)}\left(D_{m}-C\right) .
\end{gathered}
$$

It follows that

$$
D_{l(i, j)}<J(i, j) \leqq C, \quad i \in \Gamma, \quad C<j<D_{i} .
$$

The mapping

$$
(i, j) \rightarrow(I(i, j), J(i, j)) \quad\left(i \in \Gamma, C<j \leqq D_{i}\right)
$$

is 1-1. Indeed, had we $i, i^{\prime} \in \Gamma, C<j \leqq D_{i}, C<j^{\prime} \leqq D_{i}^{\prime}$,

$$
(I(i, j), J(i, j))=\left(I\left(i^{\prime}, j^{\prime}\right), J\left(i^{\prime}, j^{\prime}\right)\right),
$$

and say $i<i^{\prime}$, then we could infer from (5) that

$$
\begin{aligned}
j+ & \sum_{i<m \leqq I(i, j)} D_{m}=J(i, j)+(I(i, j)-i) C, \\
& \sum_{i^{\prime} \leqq m \leqq I(i, j)} D_{m} \geqq J(i, j)+\left(I(i, j)-i^{\prime}\right) C,
\end{aligned}
$$

and therefore that

$$
j+\sum_{i<m<i^{\prime}} D_{m} \leqq\left(i^{\prime}-i\right) C,
$$

in contradiction to $j>C$ or to (4). We define now

$$
U x=\left(\left(y_{i, j}\right)_{j=1}^{C}\right)_{i=-\infty}^{\infty} \in\left(\Omega^{C}\right)^{Z}
$$


by setting

$$
\begin{aligned}
y_{i, j}=x_{i, j}, & & \text { if } i \in Z, & 1 \leqq j \leqq \min \left(C, D_{i}\right), \\
y_{I(i, j), J(i, j)}=x_{i, j}, & & \text { if } i \in \Gamma, & C<j \leqq D_{i},
\end{aligned}
$$

and by setting $y_{i, j}=\alpha, \alpha \in \Omega, \alpha \neq \omega$, elsewhere. $U$ is Borel and it commutes with the shifts. We prove now that it is $1-1$ by showing that the $D_{i}, i \in \Gamma$, can be computed from $U x$.

Denote

$$
I_{\omega}(i)=I\left(i, D_{i}\right), \quad J_{\omega}(i)=J\left(i, D_{i}\right), \quad i \in \Gamma,
$$

and

$$
N_{\omega}(i)=\sum_{j=1}^{c} \delta_{\omega, y_{i, j}}, \quad i \in Z
$$

We have for $i, i^{\prime} \in \Gamma$

$$
\begin{aligned}
i<i^{\prime}<I_{\omega}(i) & \Rightarrow I_{\omega}\left(i^{\prime}\right) \leqq I_{\omega}(i), \\
i<I_{\omega}\left(i^{\prime}\right)<I_{\omega}(i) & \Rightarrow i<i^{\prime}, \\
i<i^{\prime}, \quad I_{\omega}(i)=I_{\omega}\left(i^{\prime}\right) & \Rightarrow J_{\omega}(i)>J_{\omega}\left(i^{\prime}\right) .
\end{aligned}
$$

From these relations and since $i \rightarrow\left(I_{\omega}(i), J_{\omega}(i)\right)(i \in \Gamma)$ is $1-1$ we have

$$
\begin{aligned}
& \sum_{i<m<I_{\omega}(i)} N_{\omega}(m)+\sum_{j=1}^{J_{\omega}^{(i)}} \delta_{\omega, y_{I_{\omega}(i), 9}} \\
& =\left|\left\{i^{\prime} \in Z-\Gamma: i<i^{\prime} \leqq I_{\omega}(i)\right\}\right|+\left|\left\{i^{\prime} \in \Gamma: i<I_{\omega}\left(i^{\prime}\right)<I_{\omega}(i)\right\}\right| \\
& \quad+\left|\left\{i^{\prime} \in \Gamma: I_{\omega}\left(i^{\prime}\right)=I_{\omega}(i), J_{\omega}\left(i^{\prime}\right)<J_{\omega}(i)\right\}\right|+1 \\
& =\left|\left\{i^{\prime} \in Z-\Gamma: i<i^{\prime} \leqq I_{\omega}(i)\right\}\right|+\left|\left\{i^{\prime} \in \Gamma: i<i^{\prime}<I_{\omega}(i)\right\}\right|+1 \\
& =I_{\omega}(i)-i+1, \quad i \in \Gamma .
\end{aligned}
$$

And we have from (7)

$$
\begin{aligned}
\sum_{i<m \leqq L} N_{\omega}(m) & =\left|\left\{i^{\prime} \in Z-\Gamma: i<i^{\prime} \leqq L\right\}\right|+\left|\left\{i^{\prime} \in \Gamma: i<I_{\omega}\left(i^{\prime}\right) \leqq L\right\}\right| \\
& =\left|\left\{i^{\prime} \in Z-\Gamma: i<i^{\prime} \leqq L\right\}\right|+\left|\left\{i^{\prime} \in \Gamma: i<i^{\prime}<L\right\}\right| \\
& \leqq L-i, \quad i \in \Gamma, \quad i<L<I_{\omega}(i) .
\end{aligned}
$$

Now we see from (9) and (10) that

$$
I_{\omega}(i)=\min \left\{L>i: \sum_{i<m \leqq L} N_{\omega}(m)>L-i\right\}, \quad i \in \Gamma
$$

and that

$$
\begin{aligned}
J_{\omega}(i)=\min \left\{1<l \leqq C: \sum_{i<m<I_{\omega}(i)}\right. & N_{\omega}(m) \\
& \left.+\sum_{j=1}^{l} \delta_{\omega, y_{I_{\omega}(i), j}}=I_{\omega}(i)-i+1\right\}, \quad i \in \Gamma .
\end{aligned}
$$


Next we observe that

$$
D_{i}=J_{\omega}(i)+\left(I_{\omega}(i)-i\right) C-\sum_{i<m \leqq I_{\omega}(i)} D_{m}, \quad i \in \Gamma .
$$

We know from (6) that

$$
i<i^{\prime}<I_{\omega}(i) \Rightarrow I_{\omega}\left(i^{\prime}\right)-i^{\prime}<I_{\omega}(i)-i, \quad i, i^{\prime} \in \Gamma .
$$

It follows that if $i_{0} \in \Gamma$ is such that

$$
I_{\omega}\left(i_{0}\right)-i_{0}=\min \left\{I_{\omega}(i)-i: i \in \Gamma\right\}
$$

then $i_{0}<i \leqq I_{\omega}\left(i_{0}\right) \Rightarrow i \in Z-\Gamma$ and we see from (13) that $D_{i_{0}}$ can be computed from the $y_{i, j}, 1 \leqq j \leqq C, i \in Z$. Finally (14) implies also that (13) can be used as a recursion formula to compute all the $D_{i}, i \in \Gamma$, from $U x$.

3. By Rohlin's result $[6,10.7]$ every ergodic i.m.p.t. is isomorphic to the shift on $N^{Z}$ together with an invariant probability measure $\mu$ such that the partition

$$
\left\{\left(n_{i}\right)_{i=-\infty}^{\infty} \in N^{Z}: n_{0}=m\right\}, \quad m \in N,
$$

has finite entropy. By part 1 of the proof there is a $C \in N$ and a 1-1 mapping

$$
n \rightarrow\left(x_{n, 1}, \ldots, x_{n}, K_{n}\right) \in \bigcup_{k=1}^{\infty}\{1,2,3\}^{k} \quad(n \in N)
$$

such that

$$
\sum_{m=1}^{\infty} K_{m} \mu\left(\left\{\left(n_{i}\right)_{i=-\infty}^{\infty} \in N^{Z}: n_{0}=m\right\}\right)<C-1
$$

We use this mapping to build a 1-1 mapping

$$
V:\left(n_{i}\right)_{i=-\infty}^{\infty} \rightarrow\left(\left(x_{n_{i}, 1}, \ldots, x_{n_{i}, K_{n}}, \omega\right)\right)_{i=-\infty}^{\infty} \in X \quad\left(\left(n_{i}\right)_{i=-\infty}^{\infty} \in N^{Z}\right)
$$

that commutes with the shifts, where we can set $\Omega=\{1,2,3, \omega\}$. The individual ergodic theorem and (15) yield

$$
\lim _{L \rightarrow \infty} \frac{1}{L} \sum_{i=1}^{L}\left(K_{n_{i}}+1-C\right)<0, \quad \text { for } \mu \text {-a.a. }\left(n_{i}\right)_{i=-\infty}^{\infty} \in N^{Z} .
$$

Hence $\mu\left(V^{-1} X_{C}\right)=1$.

By part 2 of the proof there is a 1-1 Borel mapping

$$
U: X_{C} \rightarrow \Omega^{Z}
$$

that commutes with the shifts. If we set for a Borel set $F \subset \Omega^{Z}$,

$$
\nu(F)=\mu\left(V^{-1} U^{-1} F\right),
$$

then we find that $\left(N^{z}, \mu, S\right)$ is isomorphic to $\left(\Omega^{z}, \nu, S\right)$. (If a finite Borel measure on a polish space is transported via a 1-1 Borel mapping to another polish space then the Borel mapping becomes an isomorphism between the measure space 
given by $\mu$ and the measure space that is given by the transported measure. This can be seen from the fact that every analytic subset of a polish space is measurable with respect to every finite Borel measure (see e.g. [2, §6, $\left.\mathrm{n}^{\circ} 9\right]$ ). Q.E.D.

3. An approximation theorem for shift-invariant measures. Let $\Omega$ be a state space containing a finite number $n$ of elements, $n \geqq 2$. We define for a probability measure $\mu$ on $\Omega^{I}, I \in N$, such that $\mu(a)>0$ for all $a \in \Omega^{I}$

$$
\tilde{h}(\mu)=-\sum_{a=\left(a_{i}\right)_{i=1}^{L} \in \Omega^{I}} \mu(a) \log \frac{\mu(a)}{\sum_{\alpha \in \Omega} \mu\left(\left(a_{1}, \ldots, a_{I-1}, \alpha\right)\right)} .
$$

We denote by $\mathfrak{M}_{I}, I \in N$, the set of all probability measures $\mu$ on $\Omega^{I}$ such that $\mu(a)>0, a \in \Omega^{I}$, and

$$
\begin{aligned}
\mu\left(\left\{b \in \Omega^{I}: a=\left(b_{i}\right)_{i=1}^{l}\right\}\right)=\mu\left(\left\{b \in \Omega^{I}: a=\left(b_{i+m}\right)_{i=1}^{l}\right\}\right), & \\
1 & \leqq m \leqq I-l, a \in \Omega^{l}, \quad 1 \leqq l<I .
\end{aligned}
$$

Further we set

$$
Z_{a}=\left\{x \in \Omega^{Z}: a=\left(x_{i}\right)_{i=1}^{I}\right\}, \quad a \in \Omega^{I}, \quad I \in N .
$$

For $\mu \in \mathfrak{M}_{I}, I \in N$, we define a shift-invariant probability measure $\hat{\mu}$ on $\Omega^{Z}$ by

$$
\begin{aligned}
\hat{\mu}\left(Z_{a}\right) & =\mu(a), \quad a \in \Omega^{I}, \\
\hat{\mu}\left(Z_{\left(\alpha_{j}\right)_{j=1}^{J}}\right) & =\frac{\hat{\mu}\left(Z_{\left(\alpha_{j}\right)_{j=2}^{J}}\right) \hat{\mu}\left(Z_{\left(\alpha_{j}\right)_{j=1}^{J-1}}\right)}{\hat{\mu}\left(Z_{\left(\alpha_{j}\right)_{j=2}^{J=1}}\right)}, \quad\left(\alpha_{j}\right)_{j=1}^{J} \in \Omega^{J}, \quad J>I .
\end{aligned}
$$

We note that (see $[6,5.10]) h(\hat{\mu})=\tilde{h}(\mu), \mu \in \mathfrak{M}_{I}, I \in N$, and that the $\hat{\mu}$ are ergodic. Indeed, the systems $\left(\hat{\mu}, S^{I}\right), \mu \in \mathfrak{M}_{I}$, arise from indecomposable Markov chains. For probability measures $\mu, \nu$ on $\Omega^{I}, I \in N$, we use the metric

$$
|\mu, \nu|=\max _{a \in \Omega^{I}}|\mu(a)-\nu(a)| \text {. }
$$

Let $I, N \in N, I<N$. We define for $x \in \Omega^{N+I-1}$ a probability measure $\lambda_{x}^{(I)}$ on $\Omega^{I}$ by

$$
\lambda_{x}^{(I)}(a)=N^{-1} \sum_{j=1}^{N} \delta_{a,\left(x_{j+i-1}\right)_{i=1}^{I}}, \quad a \in \Omega^{I} .
$$

We set also

$$
A(I, \mu, \delta, N)=\left\{x \in \Omega^{N+I-1}:\left|\lambda_{x}^{(I)}, \mu\right|<\delta\right\}, \quad \mu \in \mathfrak{M}_{I}, \quad \delta>0 .
$$

(3.1) Lemma. Let $\mu \in \mathfrak{M}_{I}, I \in N$ and let $\varepsilon, \delta>0$. Then there is an $L>I$ such that

$$
|A(I, \mu, \delta, N)|>\exp [(\tilde{h}(\mu)-\varepsilon) N], \quad N \geqq L .
$$

Proof. The mean ergodic theorem and the Shannon-McMillan theorem [1, p. 129] show that there is an $L \in N$ such that for all $N \geqq L$

$\hat{\mu}\left(\left\{x \in \Omega^{Z}:\left|\lambda_{\left(x_{i}\right)_{i=1}^{N+1-1}}^{(I)}, \mu\right|<\delta\right\}\right.$

$$
\left.\cap\left\{x \in \Omega^{Z}:\left|N^{-1} \log \hat{\mu}\left(Z_{\left(x_{i}\right)_{i=1}^{N+1}+1}\right)+\tilde{h}(\mu)\right|<\varepsilon\right\}\right)>e^{-\varepsilon} .
$$


We infer from this that

$$
|A(I, \mu, \delta, N)|>\exp [(\tilde{h}(\mu)-\varepsilon) N-\varepsilon], \quad N \geqq L .
$$

Q.E.D.

We say that an $a \in \Omega^{l}, l \in N$, is a coding sequence if

$$
\left(a_{i}\right)_{i=1}^{m} \neq\left(a_{l-m+i}\right)_{i=1}^{m}, \quad 1 \leqq m<l .
$$

We say that an $a \in \Omega^{l}$ is an $\alpha$-coding sequence of length $l$ if $a_{i}=\alpha, 1 \leqq i<l$ and $a_{l}=\beta \neq \alpha$. We set for $I, N \in N, I<N, \mu \in \mathfrak{M}_{I}$ and $\delta>0$,

$$
B_{a}(I, \mu, \delta, N)=\left\{x \in A(I, \mu, \delta, N):\left(x_{m+i}\right)_{i=1}^{l} \neq a, 0 \leqq m \leqq N-I+1-l\right\} .
$$

(3.2) Lemma. Let $\mu \in \mathfrak{M}_{I}, I \in N$, and let $\delta, \varepsilon>0$. Then there exists a $K \in N$ with the following property: For all $\alpha$-coding sequences a of length $L \geqq K$

$$
\left|B_{a}(I, \mu, \delta, N)\right|>\exp [(\tilde{h}(\mu)-\varepsilon) N], \quad N \geqq L .
$$

Proof. By (3.1) we can find an $M \in N$ such that

$$
|A(I, \mu, \delta / 2, N)|>\exp [(\tilde{h}(\mu)-\varepsilon) N], \quad N \geqq M .
$$

We claim that any $K \in \boldsymbol{N}$ such that

$$
K>4(M+I) \varepsilon^{-1} \delta^{-1} n
$$

has the property that is stated in the lemma. Indeed, if $a$ is an $\alpha$-coding sequence of length $L$ then with $\beta \neq \alpha$

$$
\begin{aligned}
B_{a}(I, \mu, \delta, N) \supset & A(I, \mu, \delta, N) \\
& \cap\left\{x \in \Omega^{N-I+1}: x_{k(L-2)}=\beta, 1 \leqq k \leqq(N+I-1)(L-2)^{-1}\right\} .
\end{aligned}
$$

If $L \geqq K$, then $L-2-I>M$. Hence, by (1), (2) and (3),

$$
\begin{aligned}
\left|B_{a}(I, \mu, \delta, N)\right| & >\exp \left[(\tilde{h}(\mu)-\varepsilon)\left(1-\varepsilon \tilde{h}(\mu)^{-1}\right) N\right] \\
& >\exp [(\tilde{h}(\mu)-2 \varepsilon) N] .
\end{aligned}
$$

We set for $I, N \in N$

$$
\Re(I, N)=\left\{k=\left(k_{a}\right)_{a \in \Omega^{I}} \in Z^{n^{I}}: k_{a}>0, a \in \Omega^{I}, \sum_{a \in \Omega^{I}} k_{a}=N\right\},
$$

and for $k \in \Re(I, N)$

$$
\begin{gathered}
\tilde{h}(k)=\tilde{h}\left(\left(N^{-1} k_{a}\right)_{\left.a \in \Omega^{I}\right),}\right. \\
C(I, N, k)=\left\{x \in \Omega^{N+I-1}: k_{a}=N \lambda_{x}^{(I)}(a), a \in \Omega^{I}\right\} .
\end{gathered}
$$

(3.3) Lemma. For all $k \in \mathfrak{R}(I, N)$

$$
|C(I, N, k)|<\exp (\tilde{h}(k) N) \prod_{a \in \Omega^{I}}\left(\frac{N}{k_{a}}\right)^{1 / 2} .
$$


Proof. It is

$$
\begin{aligned}
|C(I, N, k)| & \leqq n^{I-1} \prod_{a \in \Omega^{I-1}} \frac{\left(\sum_{\alpha \in \Omega} k_{\left(a_{1}, \ldots, a_{I-1}, \alpha\right)}\right) !}{\prod_{\alpha \in \Omega} k_{\left(a_{1}, \ldots, a_{I-1}, \alpha\right)} !} \\
& =n^{I-1} \prod_{a \in \Omega^{I-1}}\left(\sum_{\alpha \in \Omega} k_{\left(a_{1}, \ldots, a_{I-1}, \alpha\right)}\right) !\left(\prod_{b \in \Omega^{I}} k_{b} !\right)^{-1} .
\end{aligned}
$$

The lemma follows from this by an application of Stirling's formula. Q.E.D.

Denote

$X_{a}=\left\{x \in \Omega^{Z}: S^{i} x \in Z_{a}, S^{-j} x \in Z_{a}\right.$, for infinitely many $\left.i, j \in N\right\}, \quad a \in \Omega^{I}, I \in N$.

(3.4) THEOREM. Let $\mu$ be an ergodic shift-invariant probability measure on $\Omega^{Z}$ such that

$$
\mu\left(Z_{a}\right)>0, \quad a \in \Omega^{I}, \quad I \in N,
$$

and let $\nu \in \mathfrak{M}_{I}, I \in N, \tilde{h}(\nu) \geqq h(\mu)$. Let $\varepsilon>0$. Then there exist coding sequences $b$ and $c$ and a homeomorphism $U: X_{b} \rightarrow X_{c}$ that commutes with the shift, such that

$$
\left|\mu\left(U^{-1} Z_{a}\right)-\nu(a)\right|<\varepsilon, \quad a \in \Omega^{I} .
$$

Proof. We remark first that we can restrict attention to the case $h(\mu)<\tilde{h}(\nu)$. Indeed, if $h(\mu)=\log n$, then

$$
\mu\left(Z_{a}\right)=\nu(a), \quad a \in \Omega^{I},
$$

and if $h(\mu)=\tilde{h}(\nu)<\log n$, then there is a $\nu^{\prime} \in \mathfrak{M}_{I}$ such that

$$
h(\mu)<\tilde{h}\left(\nu^{\prime}\right) \text {, }
$$

and $\left|\nu^{\prime}(a)-\nu(a)\right|<\varepsilon / 2, a \in \Omega^{I}$.

Let therefore

$$
4 \xi=\tilde{h}(\nu)-h(\mu)>0 .
$$

We choose an $I^{\prime} \geqq I$ such that $\tilde{h}(\nu)-\tilde{h}\left(\mu^{\prime}\right)<\xi$, where $\mu^{\prime}(a)=\mu\left(Z_{a}\right), a \in \Omega^{I^{\prime}}$. Let

$$
6 \varepsilon^{\prime}=n^{I-I^{\prime}} \varepsilon
$$

Let also

$$
\nu^{\prime}(a)=\hat{v}\left(Z_{a}\right), \quad a \in \Omega^{I^{\prime}}
$$

We set $2 \delta=\min _{a \in \Omega^{I^{\prime}}} \mu\left(Z_{a}\right)$ and

$$
F_{N}=\left\{x \in \Omega^{N+I^{\prime}-1}: h\left(\lambda_{x}^{\left(I^{\prime}\right)}\right)-h(\mu)<2 \xi, \min _{a \in \Omega^{I^{\prime}}} \lambda_{x}^{\left(I^{\prime}\right)}(a)>\delta\right\}, \quad N>I^{\prime} .
$$

As a consequence of the individual ergodic theorem there is an $M \in N$ such that

$$
\mu\left(\bigcap_{M^{\prime},-M^{\prime \prime} \geqq M}\left\{x \in \Omega^{Z}:\left(x_{i}\right)_{i=M^{\mu}}^{M^{\prime}} \in F_{M^{\prime}-M^{*}-I^{\prime}+2}\right\}\right)>1-\varepsilon^{\prime} .
$$


By (3.2) we can also find an $L \in N, L \geqq n$, such that for all $\gamma$-coding sequences $c$ of length $L^{\prime} \geqq L$

$$
\left|B_{c}\left(I^{\prime}, \nu^{\prime}, \varepsilon^{\prime}, N\right)\right|>\exp [(h(v)-\xi) N], \quad N \geqq L^{\prime} .
$$

Let further $J \in N, J>\xi^{-1}$, be such that

$$
J^{-n^{I^{\prime}}} \delta^{\left(n^{I^{\prime}} / 2\right)} \exp (J \xi)>1 .
$$

We choose now a $K \geqq L$ and $\alpha_{1}, \ldots, \alpha_{K} \in \Omega$ such that

$$
\left(I^{\prime}+M+J+K\right) n^{-K}<\varepsilon^{\prime},
$$

and

$$
\mu\left(Z_{\left(\alpha_{i}\right)_{i=1}^{K}}\right)<n^{-K} .
$$

Let $b \in \Omega^{2 K+1}$ be the coding sequence that is given by

$$
\begin{aligned}
b_{k} & =\alpha_{k}, & & \text { if } 1 \leqq k \leqq K, \\
& =\alpha_{1}, & & \text { if } k=K+1, \\
& =\gamma \neq \alpha_{1}, & & \text { if } K+1<k \leqq 2 K+1,
\end{aligned}
$$

and set

$$
Y=\Omega^{Z}-\bigcup_{i=1}^{2 K+1} S^{i} Z_{b}
$$

We have from (8) and (9)

$$
\mu(Y)>1-2 \varepsilon^{\prime}
$$

We define for $x \in Y$

$$
i^{+}(x)=\min \left\{i \geqq 0: S^{i} x \in Z_{b}\right\}, \quad i^{-}(x)=\min \left\{i \geqq 0: S^{-i-2 K-2} \in Z_{b}\right\} .
$$

(3.3) together with (6) and (7) implies that for a $\gamma$-cocing sequence $c$ of length $2 K+1$

$$
\begin{aligned}
& \left|B_{c}\left(I^{\prime}, \nu^{\prime}, \varepsilon^{\prime}, N\right)\right|\left|F_{N}\right|^{-1}>\exp [(\tilde{h}(\nu)-\xi) N] N^{-n^{I^{\prime}}} \delta^{\left(n^{\prime} / 2\right)} \exp [-(h(\mu)+2 \xi) N] \\
& =N^{-n^{\prime}} \delta^{\left(n^{l^{\prime}} / 2\right)} \exp (\xi N)>1, \quad N \geqq J+2 K+1 \text {. }
\end{aligned}
$$

We see now that there are mappings $\varphi_{N}, N \in N$, of $\Omega^{N}$ onto itself such that

$$
\begin{array}{r}
\varphi_{N+I^{\prime}-1}\left(\left\{a \in F_{N}:\left(a_{i}\right)_{i=l}^{l+2 R} \neq b, 1 \leqq l \leqq N+I^{\prime}-2 K\right\}\right) \subset B_{c}\left(I^{\prime}, \nu^{\prime}, \varepsilon^{\prime}, N\right), \\
N \geqq J+2 K+1,
\end{array}
$$

and such that

$$
\begin{aligned}
\varphi_{N}\left(\left\{a \in \Omega^{N}:\left(a_{i}\right)_{i=l}^{l+2 K}\right.\right. & \neq b, 1 \leqq l \leqq N-2 K\}) \\
& =\left\{a \in \Omega^{N}:\left(a_{i}\right)_{i=l}^{l+2 K} \neq c, 1 \leqq l \leqq N-2 K\right\}, \quad N \geqq 2 K+1 .
\end{aligned}
$$


We define now a homeomorphism $U: X_{b} \rightarrow X_{c}$, that commutes with $S$ by setting for $x \in Z_{b} \cap X_{b}, U x=y$, where

$$
\begin{gathered}
y_{i}=c_{i}, \quad 1 \leqq i \leqq 2 K+1 \\
\left(y_{i}\right)_{i=-i^{-}(x)}^{0}=\varphi_{i^{-}(x)+1}\left(x_{i}\right)_{i=-i^{-}(x)}^{0} .
\end{gathered}
$$

To conclude the proof of the theorem we use (5), (8), (9) and (10) to get

$$
\begin{aligned}
\mu\left(\left\{x \in Y: i^{+}(x)+i^{-}(x) \geqq J+2 K\right.\right. & \left.\left., i^{+}(x) \geqq I^{\prime}-1,\left(x_{i}\right)_{i=-i-(x)}^{i^{+}(x)} \in F_{i^{+}(x)+i^{-}(x)-I^{\prime}+2}\right\}\right) \\
& >\mu\left(\left\{x \in Y: i^{+}(x), i^{-}(x) \geqq I^{\prime}+M+J+K\right\}\right)-\varepsilon^{\prime} \\
& >1-2\left(I^{\prime}+M+J+K\right) n^{-K}-3 \varepsilon^{\prime} \\
& >1-5 \varepsilon^{\prime} .
\end{aligned}
$$

We infer from this by applying the individual ergodic theorem that

$$
\left(\bar{\nu}^{\prime}(a)-\varepsilon^{\prime}\right)\left(1-5 \varepsilon^{\prime}\right)<\mu\left(U^{-1} Z_{a}\right)<\nu^{\prime}(a)+6 \varepsilon^{\prime}, \quad a \in \Omega^{I^{\prime}} .
$$

Finally by (4)

$$
\left|\mu\left(U^{-1} Z_{a}\right)-\nu(a)\right|<\varepsilon, \quad a \in \Omega^{I} .
$$

We want to point out the following consequence of (3.4). Let

$$
X=\bigcap_{I=1}^{\infty} \bigcap_{a \in \Omega^{I}} X_{a},
$$

and let $\mathfrak{M}_{h}$ be the set of shift-invariant ergodic probability measures $\mu$ on $\Omega^{Z}$ such that $\mu\left(Z_{a}\right)>0, a \in \Omega^{I}, I \in N$, and $\mu(X)=1, h(\mu)=h, 0 \leqq h \leqq \ln n$.

The $\mathfrak{M}_{h}$ with the weak topology are polish spaces. The group \&S of homeomorphisms of $X$ that commute with the shift acts on $\mathfrak{M}_{h}$ by $\mu \rightarrow U \mu, \mu \in \mathfrak{M}_{h}$, $U \in$ BS, where

$$
U \mu\left(Z_{a}\right)=\mu\left(U^{-1} Z_{a}\right), \quad a \in \Omega^{I}, \quad I \in N .
$$

The homeomorphism that we have constructed in the proof of (3.4) maps $X$ onto $X$. It follows therefore from (3.4) that the transformation groups $\left(\mathfrak{S}, \mathfrak{M}_{h}\right)$ are minimal, $0 \leqq h \leqq \ln n$.

\section{An estimate for $\Delta$.}

(4.1) LemMA. For every ergodic shift-invariant probability measure $\mu$ on $\Omega^{Z}$ there exists a shift-invariant probability measure $\nu$ on $\Omega^{Z}$ such that for all $a \in \Omega^{I}$, $I \in N, v\left(Z_{a}\right)>0$, and such that the systems $\left(\Omega^{Z}, \mu, S\right)$ and $\left(\Omega^{Z}, \nu, S\right)$ are isomorphic.

Proof. If there is a $d \in \bigcup_{I=1}^{\infty} \Omega^{I}$, such that $\mu\left(Z_{d}\right)=0$, then we can assign in a 1-1 manner to every $a \in \bigcup_{I=1}^{\infty} \Omega^{I}$ a coding sequence $b(a)$ that contains $a$ as a subsequence such that $\mu\left(Z_{b(a)}\right)=0$. Let $L(a)$ be the length of $b(a)$. We can find Borel sets $A_{a} \subset \Omega^{Z}$ such that for all $a, a^{\prime} \in \bigcup_{I=1}^{\infty} \Omega^{I}$

$$
\mu\left(S^{l} A_{a} \cap S^{l^{\prime}} A_{a}\right)=0, \quad 0 \leqq l, \quad l^{\prime} \leqq 2 L(a),
$$


and

$$
\mu\left(\left(\bigcup_{l=0}^{2 L(a)} S^{l} A_{a}\right) \cap\left(\bigcup_{l=0}^{2 L\left(a^{\prime}\right)} S^{l^{\prime}} A_{a^{\prime}}\right)\right)=0
$$

Choose $c(a) \in \Omega^{L(a)}$ such that $\mu\left(Z_{c(a)} \cap A_{a}\right)>0$.

A Borel mapping $U: \Omega^{Z} \rightarrow \Omega^{Z}$ that commutes with the shift can be defined by

$$
(U x)_{i}=x_{i}, \quad \text { if } S^{i} x \notin \bigcup_{I=1}^{\infty} \bigcup_{a \in \Omega^{I}}\left(Z_{c(a)} \cap A_{a}\right),
$$

and

$$
U x \in Z_{c(a)}, \quad \text { if } x \in Z_{c(a)} \cap A_{a}, \quad a \in \Omega^{I}, \quad I \in N .
$$

Setting for a Borel set $F \in \Omega^{Z}, \nu(F)=\mu\left(U^{-1} F\right)$ proves the lemma. Q.E.D.

(4.2) LeMmA. Let $T$ be an ergodic i.m.p.t. of $(E, \mathfrak{B}, p)$ with a generator

$$
\left\{A_{0}, \ldots, A_{m}\right\}, \quad m>1,
$$

such that

$$
p\left(A_{0}\right)>p\left(A_{1}\right)+2 p\left(A_{2}\right) .
$$

Then $\Delta(T) \leqq m$.

Proof. This lemma follows from a slightly generalized version of a theorem of A. H. ZaslavskiI [7, p. 295]. Q.E.D.

(4.3) THEOREM. Let $T$ be an ergodic i.m.p.t. Then $\Delta(T) \leqq e^{h(T)}+1$.

Proof. By (2.1) there exist a state space $\Omega=\{0, \ldots, m\}, m \in N$, and a shiftinvariant probability measure $\mu$ on $\Omega^{Z}$ such that $T$ is isomorphic to the system $\left(\Omega^{Z}, \mu, S\right)$. By (4.1) we can assume here that $\mu\left(Z_{a}\right)>0, a \in \Omega^{I}, I \in N$. If now $m>e^{h(T)}$, then we can find a $q, 0<q<(2 m)^{-1}$ such that

$$
h\left(\left(\lambda_{k}\right)_{k=0}^{m}\right)>h(T),
$$

where

$$
\lambda_{0}=n^{-1}+q, \lambda_{1}=n^{-1}-2 q, \lambda_{2}=q, \lambda_{k}=n^{-1}, \quad 2<k \leqq m .
$$

From (3.4) we see now that there is a shift-invariant probability measure $\nu$ on $\Omega^{Z}$ such that $\left(\Omega^{z}, \mu, S\right)$ is isomorphic to $\left(\Omega^{z}, \nu, S\right)$ and such that

$$
\left|\nu\left(Z_{(k)}\right)-\lambda_{k}\right|<q / 4, \quad 1 \leqq k \leqq m .
$$

It is then

$$
v\left(Z_{(0)}\right)>v\left(Z_{(1)}\right)+2 v\left(Z_{(2)}\right)
$$

and the theorem follows by means of (4.2). Q.E.D.

(4.4) COROLlary. Let $T$ be the cartesian product of the $n$-shift with entropy $\ln n, n \geqq 2$, and an ergodic i.m.p.t. with entropy zero. Then $\Delta(T)=n+1$. 


\section{REFERENCES}

1. P. Billingsley, Ergodic theory and information, Wiley, New York, 1965. MR 33 \#254.

2. N. Bourbaki, Topologie générale, 2ème éd., Livre III Chapitre 9: Utilisation des nombres réels en topologie générale, Actualités Sci. Indust., no. 1045, Hermann, Paris, 1958. MR 30 \#3439.

3. F. Hahn and W. Parry, Some characteristic properties of dynamical systems with quasidiscrete spectra, Math. Systems Theory 2 (1968), 179-190. MR 37 \#6435.

4. K. Jacobs, Lecture notes on ergodic theory, Aarhus Universitet, 1962/63.

5. W. Parry, Entropy and generators in ergodic theory, Benjamin, New York, 1969.

6. V. A. Rohlin, Lectures on the entropy theory of transformations with invariant measure, Uspehi Mat. Nauk 22 (1967), no. 5 (137), 3-56= Russian Math. Surveys 22 (1967), no. 5, 1-52. MR 36 \#349.

7. A. H. Zaslavski1, On the isomorphism problem for stationary processes, Teor. Verojatnost i Primenen. 9 (1964), 318-326= Theor. Probability Appl. 9 (1964), 291-298. MR 29 \#1674.

Ohio State University,

Columbus, Оhо 43210 